Sādhanā Vol. 37, Part 1, February 2012, pp. 17-31. (C) Indian Academy of Sciences

\title{
Size effect on strength and lifetime probability distributions of quasibrittle structures ${ }^{\#}$
}

\author{
ZDENĚK P BAŽANT ${ }^{1, *}$ and JIA-LIANG LE ${ }^{2}$ \\ ${ }^{1}$ Department of Civil and Environmental Engineering, Northwestern University, \\ 2145 Sheridan Road, CEE/A135, Evanston, Illinois 60208, USA \\ ${ }^{2}$ Department of Civil Engineering, University of Minnesota, \\ 500 Pillsbury Drive S.E., Minneapolis, MN 55455, USA \\ e-mail: z-bazant@northwestern.edu; jle@umn.edu
}

\begin{abstract}
Engineering structures such as aircraft, bridges, dams, nuclear containments and ships, as well as computer circuits, chips and MEMS, should be designed for failure probability $<10^{-6}-10^{-7}$ per lifetime. The safety factors required to ensure it are still determined empirically, even though they represent much larger and much more uncertain corrections to deterministic calculations than do the typical errors of modern computer analysis of structures. The empirical approach is sufficient for perfectly brittle and perfectly ductile structures since the cumulative distribution function (cdf) of random strength is known, making it possible to extrapolate to the tail from the mean and variance. However, the empirical approach does not apply to structures consisting of quasibrittle materials, which are brittle materials with inhomogeneities that are not negligible compared to structure size. This paper presents a refined theory on the strength distribution of quasibrittle structures, which is based on the fracture mechanics of nanocracks propagating by activation energy controlled small jumps through the atomic lattice and an analytical model for the multi-scale transition of strength statistics. Based on the power law for creep crack growth rate and the cdf of material strength, the lifetime distribution of quasibrittle structures under constant load is derived. Both the strength and lifetime cdfs are shown to be sizeand geometry-dependent. The theory predicts intricate size effects on both the mean structural strength and lifetime, the latter being much stronger. The theory is shown to match the experimentally observed systematic deviations of strength and lifetime histograms of industrial ceramics from the Weibull distribution.
\end{abstract}

Keywords. Scaling; probabilistic mechanics; activation energy; histogram testing.

\footnotetext{
\#This paper is reprinted with minor modifications from Proceedings of IMECE 2009 ASME 2009 International Mechanical Engineering Congress \& Exposition November 13-19, 2009, Lake Buena Vista, USA. (Bažant \& Le 2009b).

*For correspondence
} 


\section{Introduction}

In the design of various engineering structures, it is of paramount importance to understand and ascertain the types of probability distributions of structural strength since an experimental verification of design strength and lifetime for the tolerable failure probability $P_{f}<10^{-6}$ (NKB 1978; Melchers 1987; Duckett 2005) is virtually impossible. For perfectly brittle structures failing at initiation of a macro-crack from one representative volume element (RVE) with a negligible size, the strength distribution is known to be Weibullian, based on the infinite weakest link model. For perfectly ductile structures, the failure load is a weighted sum of the strengths of the RVEs along the failure surface. According to the Central Limit Theorem, the strength distribution must follow the Gaussian (or normal) distribution.

It has been shown that the failure behaviour of quasibrittle structures varies from quasiplastic to perfectly brittle as the structure size increases (Bažant \& Planas 1998; Bažant 2004, 2005). Consequently, it must be expected that the type of probabilistic distribution of strength of quasibrittle structures will vary with the structure size and geometry.

This study deals with quasibrittle structures of positive geometry, which is a broad class of sturctures that fail at the macro-crack initiation from one RVE. Extensive experimental data showed that the strength histograms of various quasibrittle materials, such as concrete, industrial and dental ceramics (Salem et al 1996; Munz \& Fett 1999; Tinschert et al 2000; Lohbauer et al 2002; dos Santos et al 2003), and fiber composites (Wagner et al 1986; Wagner 1989), consistently deviate from the two-parameter Weibull distribution. Recently, the three-parameter Weibull distribution with a non-zero threshold has been adopted as a remedy (Stanley \& Inanc 1985; Duffy et al 1993; Gross 1996).

It is observed, however, that the three-parameter Weibull distribution still shows a systematic deviation from sufficiently broad experimental histograms in the high probability regime (Bažant $\&$ Pang 2007). Furthermore, the three-parameter Weibull distribution implies a vanishing size effect on the mean strength for large size structures, which does not agree with the experimental observations (Le \& Bažant 2009; Pang et al 2008). In fact, the experimental size effect curve on concrete and fiber composites (Bažant et al 2006, 2007) at large size limit implies that the threshold must be zero (Pang et al 2008).

Recent studies (Le \& Bažant 2009; Bažant \& Pang 2007) showed that the problem actually lies in the assumption of infinite weakest-link model which underlies the Weibull statistics of strength. One must consider a finite weakest-link model because of the fact that the size of one RVE is not negligible compared to the structure size, which is the salient feature of all quasibrittle structures. A probabilistic theory was recently developed to model the strength distribution of quasibrittle structures (Bažant \& Pang 2006, 2007; Bažant et al 2009).

The theory is derived from the strength statistics of a nano-structure considered as a nano-scale block of either a regular lattice or a disordered nano-structure, and is based on the probabilistic fracture mechanics of random jumps of the crack tip propagating through the nano-structure (Bažant et al 2008, 2009). The transition of strength statistics from the nano-scale to the RVE scale can be mechanically represented by a hierarchical model consisting of bundles and chains (Bažant \& Pang 2006, 2007). Based on the asymptotic properties of strength cdfs of bundles and chains, it has been shown that the strength cdf of one RVE can be approximately modelled as a Gaussian distribution onto which a power-law tail is grafted at the failure probability of about $P_{f} \approx 10^{-4}-10^{-3}$.

With the crack growth rate law, the theory is further extended to model the lifetime cdf of quasibrittle structures under constant loads. The model agrees well with the strength and lifetime 
histograms of various quasibrittle materials such as concrete, industrial and dental ceramics and fiber composites (Bažant \& Pang 2007; Pang et al 2008; Le \& Bažant 2009).

This paper, representing both a digest and an extension of Bažant et al (2009), reviews the recently developed theory, and derives some key asymptotic properties of strength distribution of bundles with softening stress-strain behaviour, which further validates the grafted distribution of RVE strength underlying the weakest-link model of structural failure. The theory is then verified by optimum fits of some recent tests of the strength and lifetime histograms of industrial ceramics.

\section{Failure statistics of nano-structure}

The failure of a structure originates from the failure of its nano-structures, either atomic lattice blocks or disordered nano-structures. Naturally, the statistics of structural failure at macro-scale must be related to the statistics of breakage of nano-structures. In the present theory, a nano-crack is considered to propagate by random jumps through either an atomic lattice block or through a disordered nano-structure. These jumps are governed by the activation energy barriers separating a series of metastable states on the surface of the free energy potential of the nano-structure.

When the nano-crack advances by one atomic spacing or one nano-inhomogeneity, the energy release due to fracture must correspond to the change of activation energy barrier. Based on the equivalent linear elastic fracture mechanics, the energy release can be explicitly related to the remote stress applied on the nano-structure (Bažant et al 2008, 2009).

Since the interatomic separation across the crack line increases by only a small fraction of the atomic spacing during each jump, the activation energy barrier for the forward jump differs very little from the backward jump. Therefore, the jumps of the state of nano-structure must be happening in both directions, though with slightly different frequencies.

By virtue of the transition rate theory (Philips 2001; Kaxiras 2003), the first-passage time for each transition can be calculated by Kramer's formula (Risken 1989), which gives the net frequency of the propagation of nano-crack. After a number of jumps of the nano-crack tip, the crack loses its stability and propagates dynamically, which leads to the break of nano-structure.

It may reasonably be assumed that each jump of the nano-crack tip is history independent (Krausz \& Krausz 1988). Therefore, the failure probability of the nano-structure is proportional to the sum of frequencies of all the jumps needed to reach a certain critical crack length. The failure probability has thus been found to follow a power-law function of the remote stress with a zero threshold (e.g., Bažant et al 2008 and Bažant et al 2009).

\section{Multi-scale transition of strength statistics}

To relate the strength cdf of an RVE at the macro-scale to the strength cdf of a nano-structure, a certain statistical multiscale transition framework is needed. Though various stochastic multiscale numerical approaches have been proposed (Graham-Brady et al 2006; Williams \& Baxer 2006; Xu 2007), the capability of these approaches is always limited due to incomplete knowledge of the uncertainties in the information across all the scales. Instead, for the sole purpose of statistics, the multi-scale transition of strength statistics has been characterized by a hierarchical model, which consists of bundles and chains shown in figure 1 (Bažant \& Pang 2006, 2007).

For a chain of $n$ elements where all of the elements have a strength cdf with a power-law tail of exponent $p$, the strength cdf of the entire chain has also a power-law tail and its exponent is 


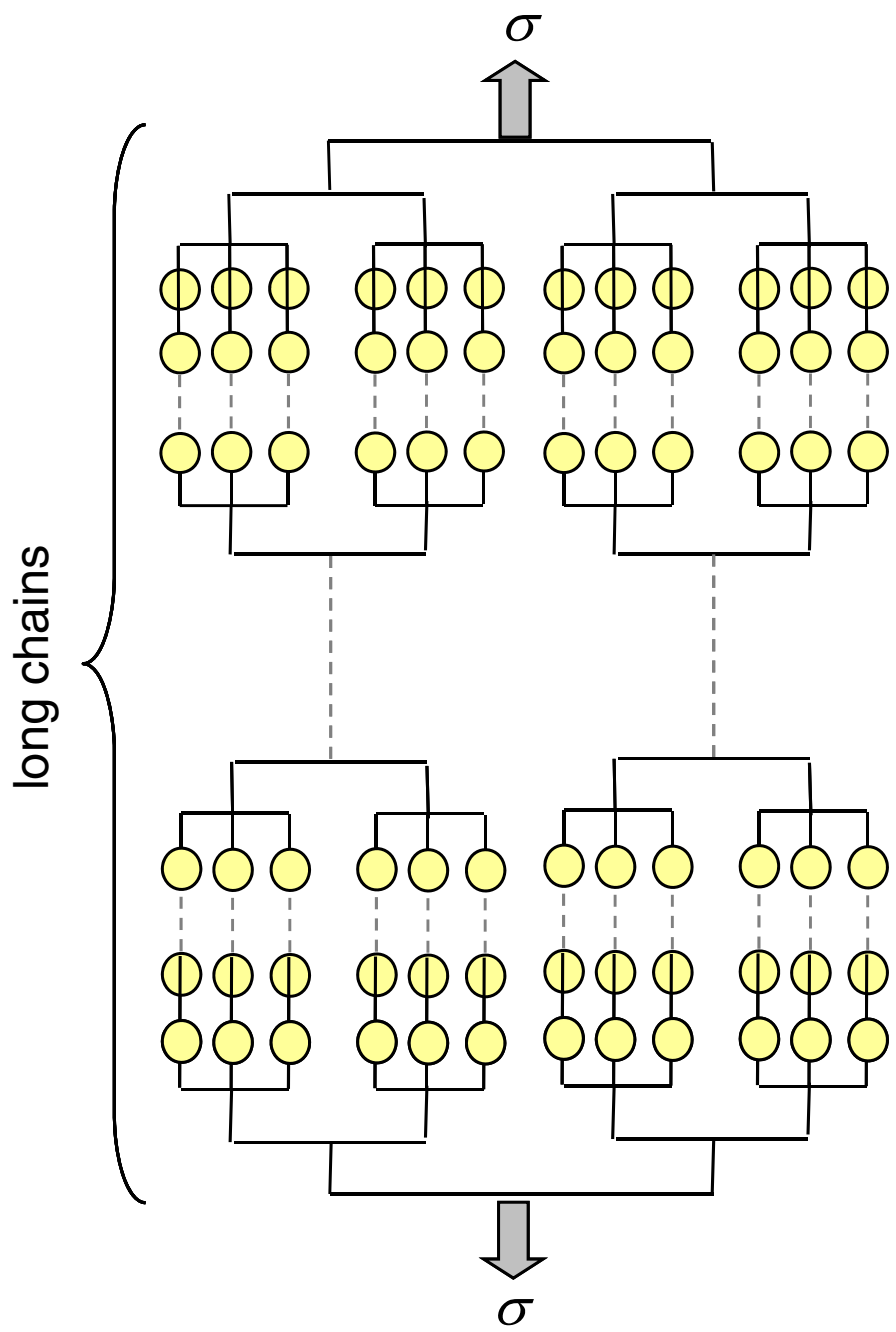

Figure 1. Hierarchical model for multiscale transition of strength statistics.

also $p$. If the tail exponents for different elements in the chain are different, then the smallest one is the tail exponent of the cdf of strength.

For a bundle of $n$ elements (or fibers) of random strength, the strength cdf of a bundle depends on the load-sharing mechanism of the fiber. Various load sharing rules have been discussed in the literature (Daniels 1945; Phoenix 1978a, b, Phoenix \& Tierney 1983; Mahesh \& Phoenix 2004). A more realistic model is to derive the load redistribution rule based on a mechanical model. Consider that all the elements (fibers) have the same elastic stiffness and are subjected to the same displacement.

Two limiting cases are now well-understood: (i) the brittle failure, in which the stress in the fiber suddenly drops to zero once it reaches the peak stress, and (ii) the plastic failure, in which, after the fiber reaches the peak stress, the stress remains constant as the displacement increases. Two asymptotic properties are of interest here: the tail behaviour of strength cdf of a bundle and the type of strength cdf for a large bundle. 
For a bundle of $n$ fibers, if the strength cdf of each fiber has a power-law tail of exponent $p_{i}(i=1, \ldots, n)$, then the strength cdf of the bundle has also a power-law tail, its exponent being $p=\sum_{i=1}^{n} p_{i}$. For the plastic bundle, this property can be simply proven by asymptotic expansion of cdf or through Laplace transform of cdf. For the brittle bundle, this property was proven by induction based on the set theory (Harlow et al 1983; Phoenix et al 1997). A simpler proof was presented in Bažant \& Pang (2007) based on asymptotic expansion of the recursive equation for the strength cdf of brittle bundle by Daniels (1945), who also showed that the cdf of a brittle bundle approaches Gaussian distribution as $n \rightarrow \infty$. For a plastic bundle, such a convergence is obvious by virtue of the Central Limit Theorem.

However, the actual behaviour of a fiber may exhibit gradual post-peak softening. For this intermediate case, the proof of additivity of tail exponents and the convergence to Gaussian distribution for a large bundle is still lacking. Here we present a simple proof.

For the tail behaviour, we first consider a bundle with two fibers of the same cross section area, though the concept applies to bundles with any number of fibers. Assume that each fiber has a bi-linear stress-strain curve (figure 2a), which has an elastic modulus $E$ and softening modulus $E_{t}\left(E_{t}=\alpha E\right)$. The only random variable in the model is the peak strength $\sigma_{i}(i=1,2)$. Then the peak stress of the bundle can be written as: $\sigma_{b}=0.5 \max _{\epsilon}\left[F_{1}(\epsilon)+F_{2}(\epsilon)\right]$, where $\epsilon=$ strain in the fiber, and $F_{1}$ and $F_{2}$ are the stresses in fibers 1 and 2, respectively. We number the two

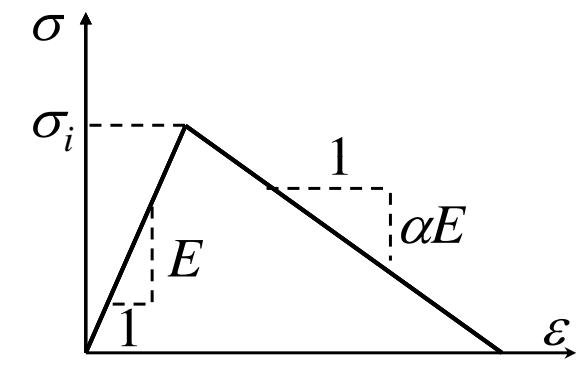

(a)

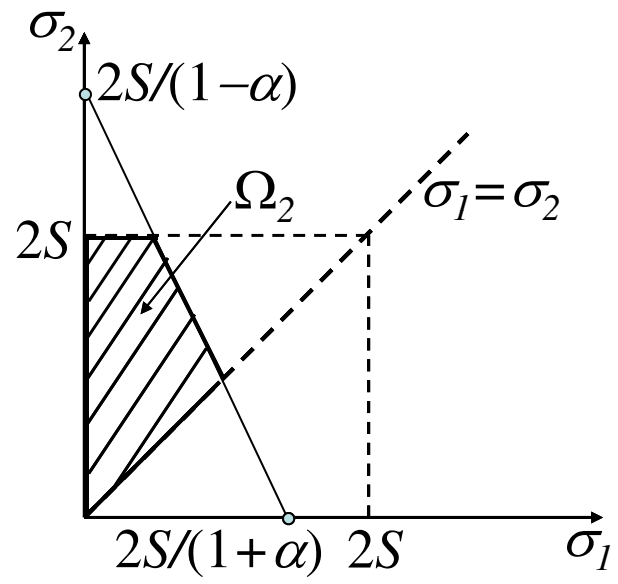

(b)

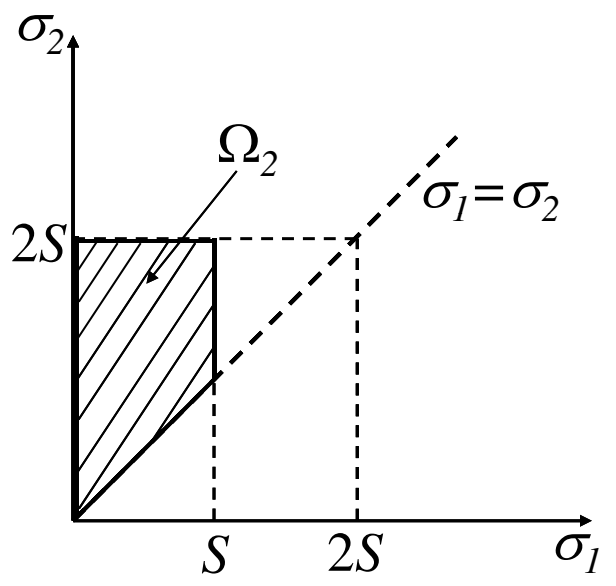

(c)

Figure 2. (a) Mechanical behaviour of softening fiber. (b) Feasible region of strength of fibers $(\alpha<1)$. (c) Feasible region of strength of fibers $(\alpha \geq 1)$. 
fibers in the order of their strengths, i.e., $\sigma_{1} \leq \sigma_{2}$. Then we can write the peak stress of the bundle, $\sigma_{b}$ as follows:

Case 1: $0 \leq \alpha \leq 1$

$$
\begin{array}{ll}
\text { if }(1+\alpha) \sigma_{1} / \alpha>\sigma_{2}: & \sigma_{b}=0.5\left[(1+\alpha) \sigma_{1}+(1-\alpha) \sigma_{2}\right] \\
\text { if }(1+\alpha) \sigma_{1} / \alpha \leq \sigma_{2}: & \sigma_{b}=0.5 \sigma_{2} .
\end{array}
$$

Case 2: $\alpha>1$

$$
\begin{array}{ll}
\text { if }(1+\alpha) \sigma_{1} / \alpha>\sigma_{2}: & \sigma_{b}=\sigma_{1} \\
\text { if }(1+\alpha) \sigma_{1} / \alpha \leq \sigma_{2}: & \sigma_{b}=\max \left(\sigma_{1}, 0.5 \sigma_{2}\right) .
\end{array}
$$

Obviously, the foregoing result covers both the plastic and brittle bundles. When $\alpha=0$, the element exhibits a plastic behaviour and the peak stress of the bundle is $0.5\left(\sigma_{1}+\sigma_{2}\right)$ (Bažant $\&$ Pang 2007). When $\alpha=\infty$, the element exhibits a brittle behaviour and the peak stress of the bundle is $\max \left(\sigma_{1}, 0.5 \sigma_{2}\right)$ (Daniels 1945).

If the strength of bundle is smaller than some prescribed value $S$, i.e., $\sigma_{b} \leq S$, then, based on Eqs. (1-4), the strength of each fiber must lie in the domain $\Omega_{2}(S)$, shown in figures $2 \mathrm{~b}$ and c. Since the strengths of these two fibers are independent random variables, the joint probability theorem indicates that the strength cdf of the bundle is:

$$
G_{2}(S)=2 \int_{\Omega_{2}(S)} f_{1}\left(\sigma_{1}\right) f_{2}\left(\sigma_{2}\right) \mathrm{d} \sigma_{1} \mathrm{~d} \sigma_{2}
$$

where $f_{i}=$ probability density function (pdf) of strength of $i$ th fiber $(i=1,2)$. Here we assume that each fiber has a strength cdf with a power-law tail, i.e., $P_{i}(\sigma)=\left(\sigma / s_{i}\right)^{p_{i}}$.

Considering the transformation: $y_{i}=\sigma_{i} / S$, the strength cdf of the bundle can be written as:

$$
G_{2}(S)=2 S^{\left(p_{1}+p_{2}\right)} \int_{\Omega_{2}(1)} \frac{p_{1} p_{2}}{s_{1}^{p_{1}} s_{2}^{p_{2}}} y_{1}^{p_{1}-1} y_{2}^{p_{2}-1} \mathrm{~d} y_{1} \mathrm{~d} y_{2},
$$

where $\Omega_{2}(1)$ is the feasible region $\Omega_{2}(S)$ normalized by $S$. Since the integral in the Eq. (6) yields a constant, the strength cdf of the bundle has a power-law tail with an exponent equal to $p_{1}+p_{2}$.

The same analysis can be applied to the bundle with $n$ fibers, where the strength cdf can be written as:

$$
\begin{aligned}
G_{n}(S) & =n ! \int_{\Omega_{n}(S)} \prod_{i=1}^{n} f_{i}\left(\sigma_{i}\right) \mathrm{d} \sigma_{1} \mathrm{~d} \sigma_{2} \ldots \mathrm{d} \sigma_{n} \\
& =n ! S^{p_{1}+p_{2}+\ldots+p_{n}} \int_{\Omega_{n}(1)}\left(\prod_{i=1}^{n} \frac{p_{i} y_{i}^{p_{i}-1}}{s_{i}^{p_{i}}}\right) \mathrm{d} y_{1} \mathrm{~d} y_{2} \ldots \mathrm{d} y_{n} .
\end{aligned}
$$

Here $\Omega_{n}(S)$ is the feasible region of stresses in all the fibers, which defines an $n$-dimensional space, and $\Omega_{n}(1)=$ is the corresponding region normalized by $S$.

Since the post-peak behaviour of each fiber only changes the geometry of $\Omega_{n}(1)$, it may be concluded that, regardless of the post-peak behaviour of each fiber, if each fiber has a strength cdf with a power-law tail, then the strength cdf of the bundle will also have a power-law tail whose exponent is equal to the sum of the exponents of the cdf tails of all the fibers. 
The reach of the power-law tail of strength cdf of a softening bundle can be estimated from Eq. (8). However, for large bundles, it is difficult to handle the integral of Eq. (8) numerically. Previous studies (Bažant \& Pang 2006, 2007) showed that the reach of power-law tail gets drastically shortened with an increasing number $n$ of elements as $P_{t n} \sim\left(P_{t 1} / n\right)^{n}-\left(P_{t 1} / 3 n\right)^{n}$ for a brittle bundle, or $\left(P_{t 1} / n\right)^{n}$ for a plastic bundle. Since the behaviour of softening bundles is bounded between these two extreme cases, the rate of shortening of power-law tail of strength cdf of the softening bundles is expected to lie between $P_{t n} \sim\left(P_{t 1} / n\right)^{n}-\left(P_{t 1} / 3 n\right)^{n}$.

To determine the type of cdf of a large bundle, one may consider a bundle of $2 n$ fibers. The force capacity of a bundle is given by $F_{\max }=\sum_{k=1}^{2 n} \sigma_{k}\left(\epsilon^{*}\right) A$, where $A=$ cross section area of each fiber, $\sigma_{k}=$ stress in $k$ th element, and $\epsilon^{*}=$ critical strain of the bundle, which leads to the maximum value of $F$. We arrange the elements according to their breaking order, $k=1,2, \ldots n$, and divide these $n$ elements into two groups: $F_{A}(\epsilon)=\sum_{i=3 k} \sigma_{i}(\epsilon) A, k=1,2, \ldots$, and $F_{B}(\epsilon)=$ $\sum_{i=3 k \pm 1} \sigma_{i}(\epsilon) A, k=0,1, \ldots$ Therefore, the maximum force of the bundle is:

$$
F_{\max }=F_{A}\left(\epsilon^{*}\right)+F_{B}\left(\epsilon^{*}\right) .
$$

If $n$ is large, then the stress distribution of elements in these two groups will be similar to that in the bundle (figure 3). It follows that the cdf of $F_{\max }$ and the cdfs of $F_{A}\left(\epsilon^{*}\right)$ and $F_{B}\left(\epsilon^{*}\right)$ are of the same type. Since $F_{A}\left(\epsilon^{*}\right) \neq F_{B}\left(\epsilon^{*}\right)$, to satisfy Eq. (9), the only possible probability distribution for $F_{\max }$ is the Gaussian distribution.

The rate of convergence, however, depends on the mechanical behaviour of each element. For the brittle bundles, the convergence is the slowest, with the error $\mathrm{O}\left(n^{-1 / 3}(\log n)^{2}\right)$. Plastic

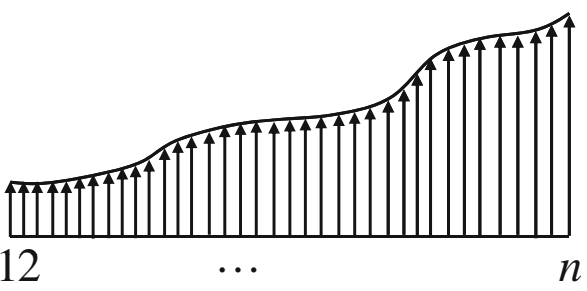

\section{Stress distribution in the bundle}

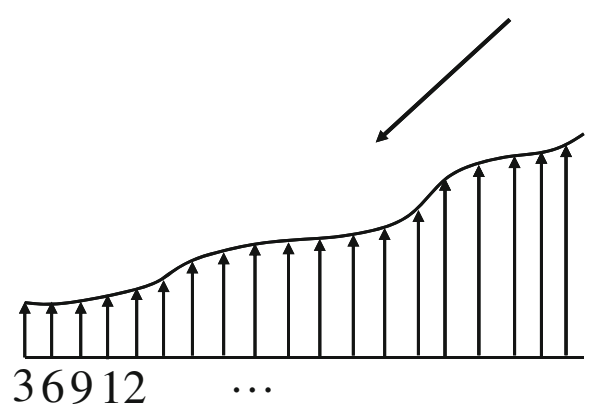

Stress distribution in sub-bundle $A$

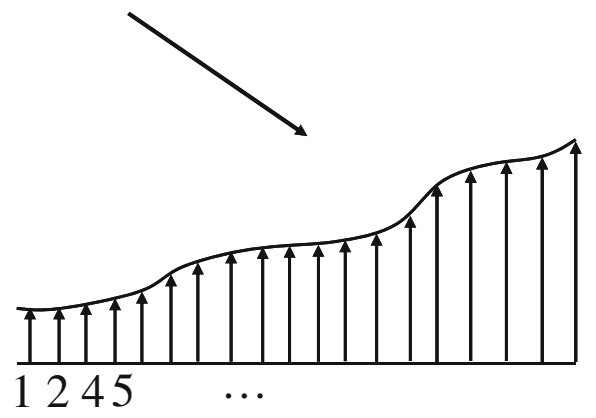

Stress distribution in sub-bundle $B$

Figure 3. Stress distribution of fibers in a large bundle. 
bundles have the fastest convergence rate, with the error $\mathrm{O}\left(n^{-1 / 2}\right)$ (according to the Central Limit Theorem) (Bažant \& Pang 2007).

To calculate the strength cdf of one RVE by the hierarchical model shown in figure 1, one must specify the mechanical behaviour of the bundles. In this model, the following assumption is introduced: for the bundles at the lowest scale, the elements (or fibers) span two rigid plates; hence they are subjected to the same deformation. For the bundles at higher scales, there can be only two fibers in such bundles (Bažant \& Pang 2007). Therefore, we may assume the simplest load-sharing rule, that is, the equal-load sharing.

As an example, we calculate the strength cdf of the hierarchical model shown in figure 1 . Every element in the hierarchical model represents one nano-structure, which has a power-law strength cdf. Three cases are considered here. They are as follows.

(i) Each element exhibits a brittle behaviour; (ii) each element exhibits a linear-softening behaviour, where the softening modulus is $40 \%$ of its elastic modulus; and (iii) each element exhibits a plastic behaviour.

Figure 4a shows the resulting strength cdf's of the hierarchical model for these three cases, on the Weibull scale. For all the cases, the lower portion of the strength cdf is a straight line on the Weibull plot which indicates that it follows the Weibull distribution (a power-law tail). Such a property is expected since the power-law tail of strength cdf is indestructible in the chain and bundle models. For the upper portion, the strength cdf deviates from the straight line.

Among the three cases, case (i) (elements with brittle behaviour) has the shortest Weibull tail, which terminates at the probability of about $5 \times 10^{-5}$. On the other hand, case (iii) (elements with plastic behaviour) has the longest Weibull tail, which terminates at the probability of about $7 \times 10^{-4}$.

To identify the upper portion of the pdf, we plot the strength cdf's in the normal distribution paper shown as figures $4 \mathrm{~b}-\mathrm{d}$. The upper portion of the cdf's can be approximately fitted by a straight line. Such an approximation is not too close for the case (i) where the cdf beyond $P_{f}=0.8$ cannot be fitted. For the cases (ii) and (iii), such an approximation can closely fit the calculated cdf's where the deviation occurs for the cdf beyond $P_{f}=0.99$, which means that the upper portion of the strength cdf can be approximated as the Gaussian distribution.

Since the nano-structure exhibits a softening behaviour, which is close to case (ii), the strength distribution of one RVE can be closely described as Gaussian, with a Weibull tail grafted on the left at the probability of about $10^{-4}-10^{-3}$. Mathematically, one may approximate the strength distribution of one RVE as Bažant \& Pang (2006, 2007).

$$
\begin{aligned}
& P_{1}\left(\sigma_{N}\right)=1-\exp \left[-\left(\sigma_{N} / s_{0}\right)^{m}\right] \quad\left(\sigma_{N} \leq \sigma_{g r}\right) \\
& P_{1}\left(\sigma_{N}\right)=P_{g r}+\frac{r_{f}}{\delta_{G} \sqrt{2 \pi}} \int_{\sigma_{g r}}^{\sigma_{N}} e^{-\left(\sigma^{\prime}-\mu_{G}\right)^{2} / 2 \delta_{G}^{2}} \mathrm{~d} \sigma^{\prime} \quad\left(\sigma_{N}>\sigma_{g r}\right),
\end{aligned}
$$

where $\sigma_{N}=$ nominal strength, which is a maximum load parameter of the dimension of stress.

In general, $\sigma_{N}=P_{\max } / b D$ or $P_{\max } / D^{2}$ for two- or three-dimensional scaling $\left(P_{\max }=\max\right.$ imum load of the structure or parameter of load system, $b=$ structure thickness in the third dimension, $D=$ characteristic structure dimension or size). Furthermore, $m$ (Weibull modulus) and $s_{0}$ are the shape and scale parameters of the Weibull tail, and $\mu_{G}$ and $\delta_{G}$ are the mean and 

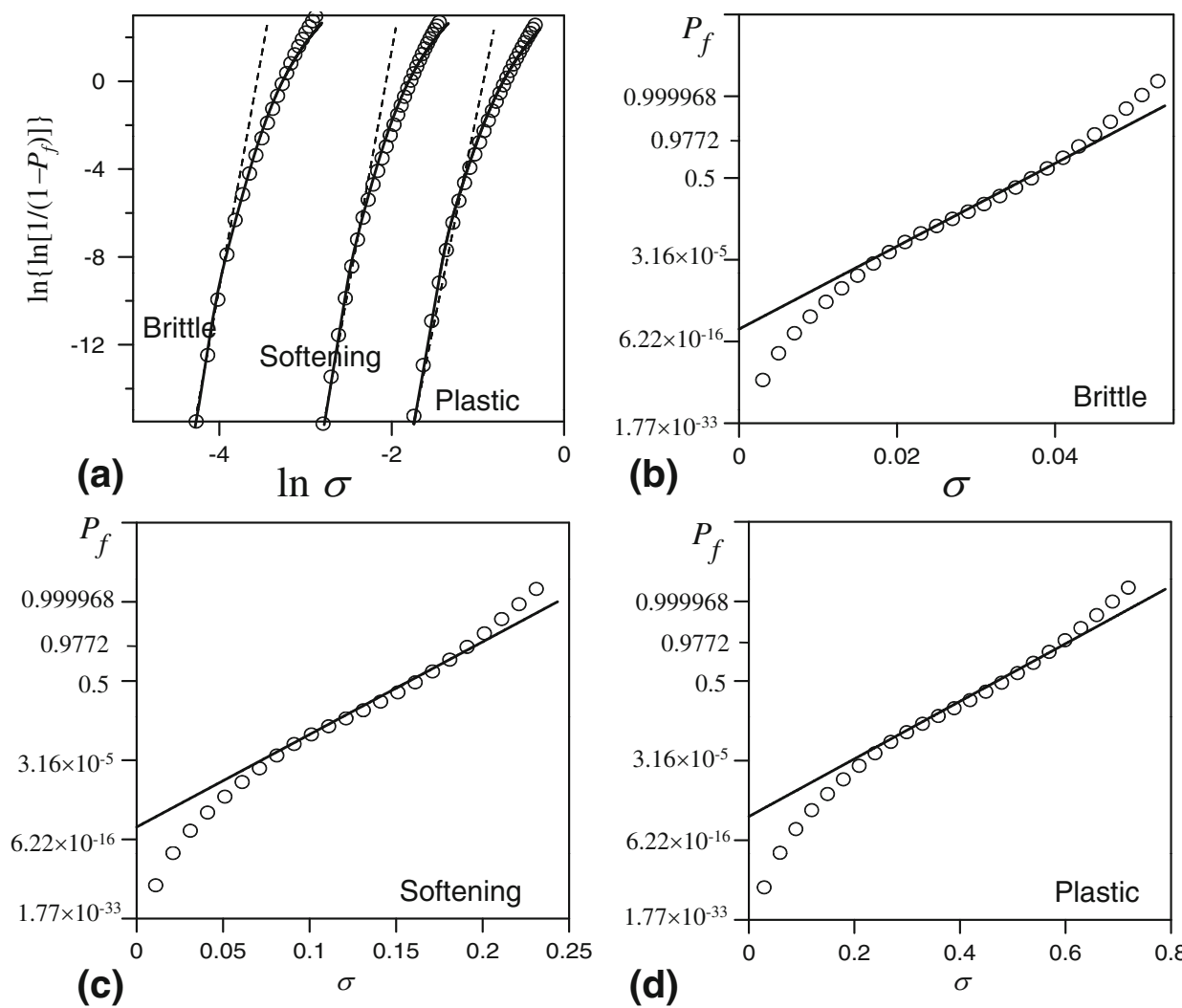

(b)

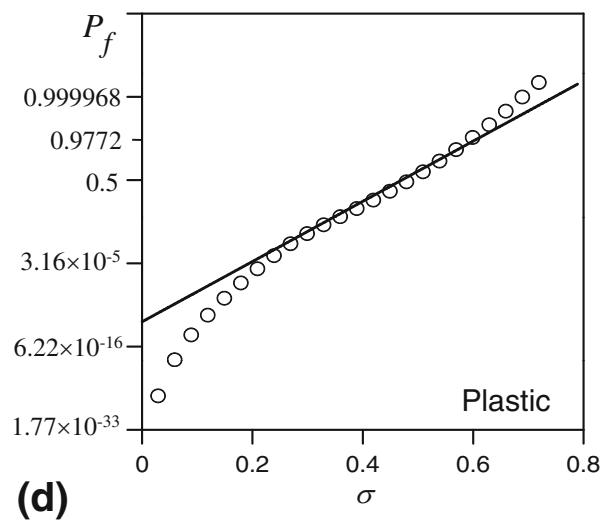

Figure 4. (a) Calculated strength cdf of one RVE on the Weibull scale. (b)-(d) Calculated strength cdf of one RVE on the normal distribution paper.

standard deviation of the Gaussian core if considered extended to $-\infty ; r_{f}$ is a scaling parameter required to normalize the grafted cdf such that $P_{1}(\infty)=1$, and $P_{g r}=$ grafting probability $=$ $1-\exp \left[-\left(\sigma_{g r} / s_{0}\right)^{m}\right]$. Finally, continuity of the probability density function at the grafting point requires that $\left.\left(\mathrm{d} P_{1} / \mathrm{d} \sigma_{N}\right)\right|_{\sigma_{g r}^{+}}=\left.\left(\mathrm{d} P_{1} / \mathrm{d} \sigma_{N}\right)\right|_{\sigma_{g r}^{-}}$.

\section{Lifetime distribution of one RVE}

It has recently been shown (Bažant et al 2009; Le et al 2009; Bažant \& Le 2009a) that one can derive the lifetime cdf of one RVE by using the power law for creep crack growth, which has been empirically described by (Evans 1972; Thouless et al 1983; Evans \& Fu 1984; Bažant \& Prat 1988; Bažant \& Jirásek 1993; Bažant \& Planas 1998; Munz \& Fett 1999)

$$
\dot{a}=C e^{-Q_{0} / k T} K^{n},
$$

where $C, n=$ empirical constant, $Q_{0}=$ activation energy, $k=$ Boltzmann's constant, $T=$ absolute temperature, $K=$ stress intensity factor. Recent studies (Bažant et al 2009; Le et al 2009) showed that, under certain plausible assumptions, the power law for creep crack growth can be physically justified on the basis of atomistic fracture mechanics and a multi-scale transition framework of fracture kinetics. 
Now consider one RVE undergoing strength and lifetime tests, where a linearly ramped load is applied in the strength test and a constant load is applied in the lifetime test. By applying Eq. (12) to these two cases, one finds the relation between the strength and lifetime of one RVE as:

$$
\sigma_{N}=\beta \sigma_{0}^{n /(n+1)} \lambda^{1 /(n+1)},
$$

where $\sigma_{N}=$ nominal strength of RVE, $\sigma_{0}=P / b D=$ applied nominal stress in the lifetime test ( $P=$ applied load), $\lambda=$ lifetime of RVE, $\beta=[r(n+1)]^{1 /(n+1)}$, and $r=$ rate of loading in the strength test. Since the distribution of RVE strength is given by Eqs. (10) and (11), the lifetime distribution of one RVE can be easily obtained by substituting Eq. (13) for $\sigma_{N}$ of Eqs. (10) and (11):

$$
\begin{aligned}
& \text { for } \lambda<\lambda_{g r}: \quad P_{1}(\lambda)=1-\exp \left[-\left(\lambda / s_{\lambda}\right)^{\bar{m}}\right] \\
& \text { for } \lambda \geq \lambda_{g r}: \quad P_{1}(\lambda)=P_{g r}+\frac{r_{f}}{\delta_{G} \sqrt{2 \pi}} \int_{\gamma \lambda_{g r}^{1 /(n+1)}}^{\gamma \lambda^{1 /(n+1)}} e^{-\left(\lambda^{\prime}-\mu_{G}\right)^{2} / 2 \delta_{G}^{2}} \mathrm{~d} \lambda^{\prime},
\end{aligned}
$$

where $\gamma=\beta \sigma_{0}^{n /(n+1)} \lambda_{g r}=\beta^{-(n+1)} \sigma_{0}^{-n} \sigma_{N, g r}^{n+1}, s_{\lambda}=s_{0}^{n+1} \beta^{-(n+1)} \sigma_{0}^{-n}$, and $\bar{m}=m /(n+1)$. Similar to the strength distribution of one RVE, the lifetime cdf of one RVE also has a Weibull tail (power-law tail). However, the rest of the lifetime cdf of one RVE does not follow the Gaussian distribution. Note that the grafting probability $P_{g r}$ for the lifetime distribution of one RVE is the same as that for the strength cdf of one RVE.

\section{Finite weakest link model and optimum fits of histograms}

To analyze softening damage and failure, the RVE must be defined as the smallest material volume whose failure triggers the failure of entire structure (Bažant \& Pang 2006; 2007). Therefore, the structure can be statistically represented by a chain of RVEs. By virtue of the joint probability theorem, and under the assumption of independence of random strengths or lifetimes of the links in a finite weakest-link model, one can calculate the strength or lifetime cdf of a structure as

$$
P_{f}(x)=1-\prod_{i=1}^{N}\left[1-P_{1}(x)\right],
$$

where $x=\sigma_{N}$ for strength distribution and $x=\lambda$ for lifetime distribution, $P_{1}=$ strength or lifetime cdf of one RVE given by Eqs. (10) and (11) or Eqs. (14) and (15).

For large size structures, what matters for $P_{f}$ is only the tail of the strength or lifetime cdf of one RVE, i.e $P_{1}=\left(\sigma_{N} / s_{0}\right)^{m}$ or $P_{1}=\left(\lambda / s_{\lambda}\right)^{m}$. By taking the logarithm of Eq. (16) and setting $\ln \left(1-P_{1}\right) \approx-P_{1}$ for small $P_{1}$, one can easily show that the strength and lifetime distributions for large size structure converge to the Weibull distribution:

$$
\begin{gathered}
P_{f}\left(\sigma_{N}\right)=1-\exp \left[-N_{e q, \sigma}\left(\sigma / s_{0}\right)^{m}\right] \\
P_{f}(\lambda)=1-\exp \left[-N_{e q, \lambda}\left(\lambda / s_{\lambda}\right)^{\bar{m}}\right],
\end{gathered}
$$

where $N_{e q, \sigma}, N_{e q, \lambda}$ are the equivalent numbers of RVEs for the strength and lifetime distributions, which can be calculated based on the elastic stress distribution in the structure (Bažant \& Pang 2007, Bažant \& Le 2009a). The equivalent number of RVE physically means that a chain that has $N_{e q, \sigma}$ or $N_{e q, \lambda}$ RVEs subjected to a uniform stress gives the same strength or lifetime 

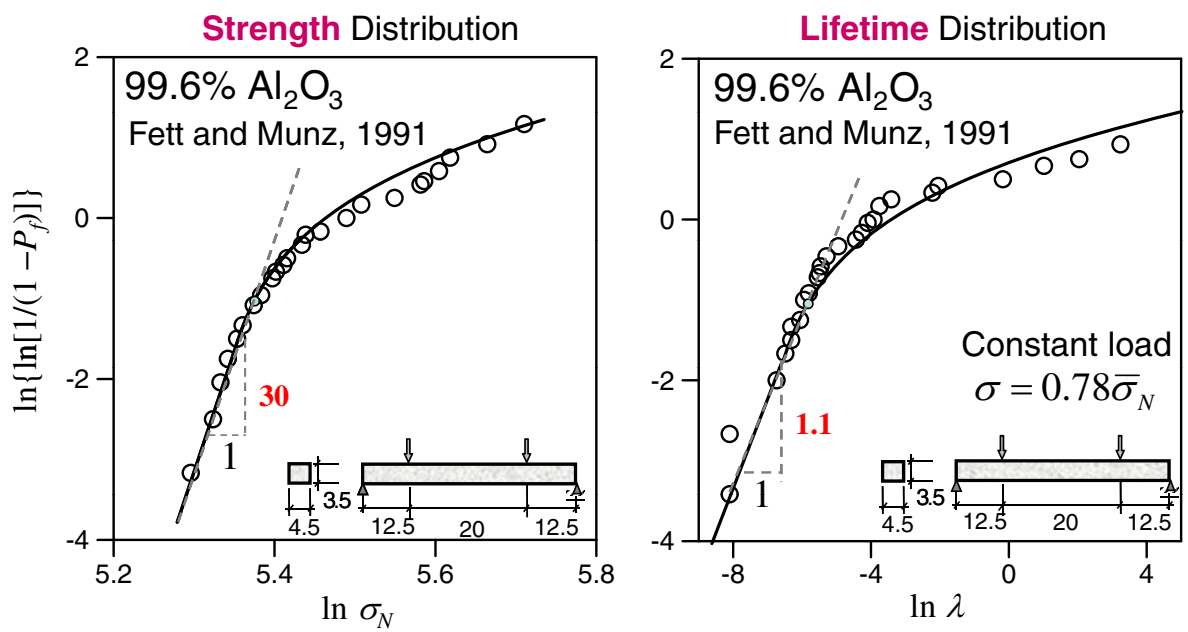

Figure 5. Optimum fits of strength and lifetime histograms of $99.9 \% \mathrm{Al}_{2} \mathrm{O}_{3}$ (Fett \& Munz 1991).

cdf as Eq. (16). The Weibull modulus of lifetime distribution is much smaller than the Weibull modulus of strength distribution. They are related by

$$
\bar{m}=m /(n+1) .
$$

Figure 5 presents the optimum fits of the strength and lifetime histograms of $99.9 \% \mathrm{Al}_{2} \mathrm{O}_{3}$ beam under four-point bend test. For each histogram, a total of 30 specimens were tested. Obviously, on the Weibull scale, both histograms do not appear to be straight lines. Instead, there is a kink separating the histogram into two parts where the lower part is a straight line and the upper part is curved. Such a pattern cannot be explained by the two-parameter Weibull distribution.

Figure 5 shows that the present theory gives excellent fits of both the strength and lifetime histograms. The location of kink actually corresponds to the grafting probability, which measures the degree of quasibrittleness of the structure.

From the data fits, it is further observed that the grafting probabilities of the strength and lifetime cdf's are about the same. This agrees well with the present theory, in which the grafting probability can be calculated as: $P_{g r}=1-\left[1-P_{g r, 1}\right]^{N_{e q}}$. Since the grafting probabilities $P_{g r, 1}$ of strength and lifetime cdfs for one RVE is the same and the equivalent number of RVE for strength cdf is almost identical to the equivalent number of RVE for lifetime cdf, then the grafting probabilities for strength and lifetime cdfs must be approximately the same.

By optimum fitting, the Weibull moduli for strength and lifetime distributions are estimated to be about 30 and 1.1, respectively. From Eq. (19), one can get exponent $n$ of the power law for creep crack growth for $99.9 \% \mathrm{Al}_{2} \mathrm{O}_{3}$, which is about 26 .

\section{Size effect on mean structural strength and lifetime}

With the grafting probability distributions of strength and lifetime of one RVE, Eq. (16) directly implies the size effects on the strength and lifetime cdf's. One can further compute the size 
effects on the mean strength and lifetime. Based on the calibrated strength and lifetime distributions of $99.9 \% \mathrm{Al}_{2} \mathrm{O}_{3}$ (figure 5), one can calculate the corresponding size effects on the mean structural strength and lifetime as shown in figure 6.

Although a close-form expression for such size effects is impossible, one can obtain the approximate form for the mean strength and lifetime by asymptotic matching. It has been proposed that the size effect on mean strength can be approximated by Bažant $(2004 ; 2005)$, Bažant \& Novák (2000c)

$$
\bar{\sigma}_{N}=\left[\frac{N_{a}}{D}+\left(\frac{N_{b}}{D}\right)^{r / m}\right]^{1 / r},
$$

where parameters $N_{a}, N_{b}, r$ and $m$ are to be determined by asymptotic properties of the size effect curve.

It has been shown that such a size effect curve agrees well with the predictions by other mechanical models such as the non-local Weibull theory (Bažant \& Novák 2000a, b), as well as with the experimental observations on concrete (Bažant et al 2007) and fiber composites (Bažant et al 2006); $m=$ Weibull modulus of the strength distribution, which can be determined by the slope of the left tail of the strength histogram plotted on the Weibull scale, or more accurately by size effect tests. The other three parameters, $N_{a}, N_{b}$, and $r$, can be determined by solving three simultaneous equations based on three asymptotic conditions, $\left[\bar{\sigma}_{N}\right]_{D \rightarrow l_{0}}$, $\left[\mathrm{d} \bar{\sigma}_{N} / \mathrm{d} D\right]_{D \rightarrow l_{0}}$, and $\left[\bar{\sigma}_{N} D^{1 / m}\right]_{D \rightarrow \infty}$, where $l_{0}=$ RVE size.

One can approximate the size effect on the mean structural lifetime by an equation with the same form as Eq. (20):

$$
\bar{\lambda}=\left[\frac{C_{a}}{D}+\left(\frac{C_{b}}{D}\right)^{r / m}\right]^{(n+1) / \varphi},
$$

where $m$ is the Weibull modulus of the cdf of strength, and $n=$ exponent of the power law for subcritical creep crack growth rate. Similar to the size effect on mean strength, $C_{a}, C_{b}$, and $\varphi$ can
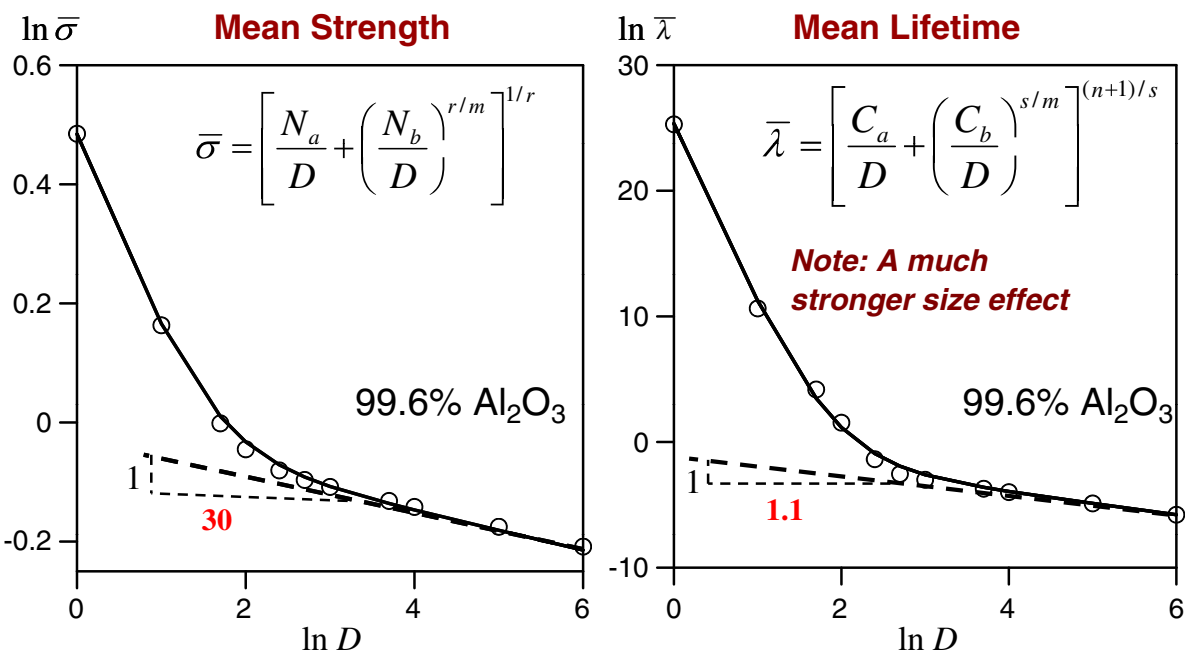

Figure 6. Calculated size effects on mean structural strength and lifetime. 
be obtained from three asymptotic conditions: $[\bar{\lambda}]_{D \rightarrow l_{0}},[\mathrm{~d} \bar{\lambda} / \mathrm{d} D]_{D \rightarrow l_{0}}$, and $\left[\bar{\lambda} D^{(n+1) / m}\right]_{D \rightarrow \infty}$. It is obvious that the size effect on the mean structural lifetime is much stronger than that on the mean strength.

\section{Conclusion}

The present theory shows that the types of strength and lifetime distributions depend on the structure size and geometry. This has significant implications for the safety factors to be used in reliability assessment for the design of many engineering structures, such as large prestressed concrete bridges, large aircraft or ships made of fiber composites, and various micro- and nanoelectronic devices. The present theory indicates that the safety factors guarding against the uncertainties in strength and lifetime cannot be empirical, and cannot be constant. They must be calculated as a function of the size, geometry of structures and environment.

\section{References}

Bažant Z P 2004 Scaling theory of quasibrittle structural failure. Proc. Natl. Acad. Sci. USA 101(37): 13397-13399

Bažant Z P 2005 Scaling of structural strength. 2nd ed., London: Elsevier, 267 pp.

Bažant Z P, Jirásek M 1993 R-curve modeling of rate and size effects in quasibrittle fracture. Int. J. Fract. 62: $355-373$

Bažant Z P, Le J-L 2009a Nano-mechanics based modeling of lifetime distribution of quasibrittle structures. J. Eng. Fail. Anal. 16: 2521-2529

Bažant Z P, Le J-L 2009b Size effect on strength and lifetime distributions of quasibrittle structures. Proc., ASME 2009 Int. Mech. Eng. Congress (IMECE2009), Lake Buena Vista, Florida, pp. 1-9

Bažant Z P, Le J-L, Bazant M Z 2008 Size effect on strength and lifetime distribution of quasibrittle structures implied by interatomic bond break activation. Proc. of 17th European conference on fracture, Brno, Czech Rep, pp. 78-92

Bažant Z P, Le J-L, Bazant M Z 2009 Scaling of strength and lifetime distributions of quasibrittle structures based on atomistic fracture mechanics. Proc. Natl. Acad. Sci. USA 106(28): 11484-11489

Bažant Z P, Novák D 2000a Probabilistic nonlocal theory for quasibrittle fracture initiation and size effect. I. Theory. J. Eng. Mech. ASCE 126(2): 166-174

Bažant Z P, Novák D 2000b Probabilistic nonlocal theory for quasibrittle fracture initiation and size effect. II. Application. J. Eng. Mech. ASCE 126(2): 175-185

Bažant Z P, Novák D 2000c Energetic- statistical size effect in quasibrittle failure at crack initiation. ACI Mater. J. 97(3): 381-392

Bažant Z P, Pang S-D 2006 Mechanics based statistics of failure risk of quasibrittle structures and size effect on safety factors. Proc. Natl. Acad. Sci. USA 103(25): 9434-9439

Bažant Z P, Pang S-D 2007 Activation energy based extreme value statistics and size effect in brittle and quasibrittle fracture. J. Mech. Phys. Solids 55: 91-134

Bažant Z P, Planas J 1998 Fracture and size effect in concrete and other quasibrittle materials, Boca Raton and London: CRC Press, 616 pp.

Bažant Z P, Prat P C 1988 Effect of temperature and humidity on fracture energy of concrete. ACI Mater. J. 85-M32: 262-271

Bažant Z P, Vořechovský M, Novák D 2007 Asymptotic prediction of energetic-statistical size effect from deterministic finite element solutions. J. Eng. Mech. ASCE 128: 153-162

Bažant Z P, Xi Y 1991 Statistical size effect in quasi-brittle structures: II. Nonlocal theory. J. Eng. Mech. ASCE 117(7): 2623-2640 
Bažant Z P, Zhou Y, Daniel I M, Caner F C, Yu Q 2006 Size effect on strength of laminate-foam sandwich plates. J. Eng. Mater. Technol. ASME 128(3): 366-374

Chiao C C, Sherry R J, Hetherington N W 1977 Experimental verification of an accelerated test for predicting the lifetime of organic fiber composites. J. Comp. Mater. 11: 79-91

Daniels H E 1945 The statistical theory of the strength of bundles and threads. Proc. R. Soc. London A. 183: 405-435

dos Santos C, Strecker K, Piorino Neto F, de Macedo Silva O M, Baldacim S A and da Silva C R M 2003 Evaluation of the reliability of $\mathrm{Si}_{3} \mathrm{~N}_{4}-\mathrm{Al}_{2} \mathrm{O}_{3}-\mathrm{CTR}_{2} \mathrm{O}_{3}$ ceramics through Weibull analysis. Mater. Res. 6(4): 463-467

Duckett K 2005 Risk analysis and the acceptable probability of failure. Struct. Eng. 83(15): 25-26

Duffy S F, Powers L M, Starlinger A 1993 Reliability analysis of structural ceramic components using a three-parameter Weibull distribution. Trans. ASME J. Eng. Gas Turbines Power 115: 109-116

Evans A G 1972 A method for evaluating the time-dependent failure characteristics of brittle materials and its application to polycrystalline alumina. J. Mater. Sci. 7: 1173-1146

Evans A G, Fu Y 1984 The mechanical behaviour of alumina. In Fracture in ceramic materials, Park Ridge, NJ: Noyes Publications, pp. 56-88

Fett T, Munz D 1991 Static and cyclic fatigue of ceramic materials. In Ceramics today - tomorrow's ceramics, P Vincenzini (ed), Elsevier Science Publisher B. V., 1827-1835

Graham-Brady L L, Arwadea S R, Corrb D J, Gutiérrezc M A, Breyssed D, Grigoriue M, Zabaras N 2006 Probability and materials: from nano- to macro-scale: a summary. Prob. Eng. Mech. 21(3): 193-199

Gross B 1996 Least squares best fit method for the three parameter weibull distribution: analysis of tensile and bend specimens with volume or surface flaw failure. NASA TM-4721: 1-21

Harlow D G, Smith R L, Taylor H M 1983 Lower tail analysis of the distribution of the strength of loadsharing systems. J. Appl. Prob. 20: 358-367

Kaxiras E 2003 Atomic and electronic structure of solids. Cambridge, UK: Cambridge University Press, $700 \mathrm{pp}$.

Krausz A S, Krausz K 1988 Fracture kinetics of crack growth. Netherland: Kluwer Academic Publisher

Le J-L, Bažant Z P 2009 Finite weakest link model with zero threshold for strength distribution of dental restorative ceramics. Dent. Mater. 25(5): 641-648

Le J-L, Bažant Z P, Bazant, M Z 2009 Crack growth law and its consequences on lifetime distributions of quasibrittle structures. J. Phys. D 106: 104119(8 pp)

Lohbauer U, Petchelt A, Greil P 2002 Lifetime prediction of CAD/CAM dental ceramics. J. Biomed. Mater. Res. 63(6): 780-785

Mahesh S, Phoenix S L 2004 Lifetime distributions for unidirectional fibrous composites under creeprupture loading. Int. J. Fract. 127: 303-360

Melchers R E 1987 Structural reliability, analysis \& prediction. New York: Wiley

Munz D, Fett T 1999 Ceramics: mechanical properties, failure behavior, materials selection. Berlin: Springer-Verlag

NKB 1978 (Nordic Committee for Building Structures). Recommendation for loading and safety regulations for structural design. NKB Report, No. 36

Pang S-D, Bažant Z P, Le J-L 2008 Statistics of strength of ceramics: finite weakest link model and necessity of zero threshold. Int. J. Frac. Special Issue on 'Physical Aspects of Scaling' 154: 131-145

Philips R 2001 Crystals, defects and microstructures: modeling across scales. Cambridge, UK: Cambridge University Press, 720 pp.

Phoenix S L 1978a Stochastic strength and fatigue of fiber bundles. Int. J. Frac. 14(3): 327-344

Phoenix S L 1978b The asymptotic time to failure of a mechanical system of parallel members. SIAM J. Appl. Maths. 34(2): 227-246

Phoenix S L, Ibnabdeljalil M, Hui C-Y 1997 Size effects in the distribution for strength of brittle matrix fibrous composites. Int. J. Solids Struct. 34(5): 545-568

Phoenix S L, Tierney L-J 1983 A statistical model for the time dependent failure of unidirectional composite materials under local elastic load-sharing among fibers. Eng. Fract. Mech. 18(1): 193-215 
Risken H 1989 The Fokker-Planc equation. 2nd ed., Berlin, Heidelberg, New York: Springer Verlag, $488 \mathrm{pp}$.

Salem J A, Nemeth N N, Powers L P, Choi S R 1996 Reliability analysis of uniaxially ground brittle materials. J. Eng. Gas Turbines Power 118: 863-871

Smith R L 1982 The asymptotic distribution of the strength of a series-parallel system with equal load sharing. Ann. Probab. 10(1): 137-171

Stanley P, Inanc E Y 1985 Assessment of surface strength and bulk strength of a typical brittle material. In S Eggwertz, N C Lind (eds), Probabilistic methods. I. The mechanics of solids and structures. Berlin: Springer-Verlag, pp. 231-251

Thouless M D, Hsueh C H, Evans A G 1983 A damage model of creep crack growth in polycrystals. Acta Metall. 31(10): 1675-1687

Tinschert J, Zwez D, Marx R, Ausavice K J 2000 Structural reliability of alumina-, feldspar-, leucite-, micaand zirconia-based ceramics. J. Dent. 28: 529-535

Wagner H D 1989 Stochastic concepts in the study of size effects in the mechanical strength of highly oriented polymeric materials. J. Polym. Sci. 27: 115-149

Wagner H D, Schwartz P, Phoenix S L 1986 Lifetime statistics for single Kevlar 49 filaments in creeprupture. J. Polym. Sci. 21: 1868-1878

Weibull W 1939 The phenomenon of rupture in solids. Proc., Royal Swedish Inst. Eng. Res. 153, Stockholm, 1939, pp. 1-55

Williams T, Baxer S C 2006 A framework for stochastic mechanics. Prob. Eng. Mech. 21(3): 247-255

Xu X F 2007 A multiscale stochastic finite element method on elliptic problems involving uncertainties. Comput. Meth. Appl. Mech. Eng. 196: 2723-2736 